\title{
Camera calibration with two arbitrary coaxial circles
}

\author{
Carlo Colombo, Dario Comanducci, and Alberto Del Bimbo \\ Dipartimento di Sistemi e Informatica \\ Via S. Marta 3, 50139 Firenze, Italy \\ \{colombo, comandu, delbimbo\}@dsi.unifi.it
}

\begin{abstract}
We present an approach for camera calibration from the image of at least two circles arranged in a coaxial way. Such a geometric configuration arises in static scenes of objects with rotational symmetry or in scenes including generic objects undergoing rotational motion around a fixed axis. The approach is based on the automatic localization of a surface of revolution (SOR) in the image, and its use as a calibration artifact. The SOR can either be a real object in a static scene, or a "virtual surface" obtained by frame superposition in a rotational sequence. This provides a unified framework for calibration from single images of SORs or from turntable sequences. Both the internal and external calibration parameters (square pixels model) are obtained from two or more imaged cross sections of the SOR, whose apparent contour is also exploited to obtain a better calibration accuracy. Experimental results show that this calibration approach is accurate enough for several vision applications, encompassing $3 \mathrm{D}$ realistic model acquisition from single images, and desktop 3D object scanning.
\end{abstract}

\section{Introduction}

Camera calibration is a fundamental problem in computer vision and photogrammetry, whose solution allows relating 2D image coordinates to directions in the 3D space. The calibration methods proposed in the literature exhibit a trade-off between geometric accuracy and flexibility of use. Very high accuracies are typically required for laboratory applications, and obtained with special purpose 3D calibration patterns [1]. On the other hand, results from projective geometry were recently used to develop flexible and reasonably accurate calibration approaches for desktop vision applications exploiting scene constraints. A popular scene-based calibration approach uses the vanishing points of three mutually orthogonal directions [2], thus proving useful in the reconstruction of architectural environments [3], [4]. Images of spheres were used for desktop calibration purposes first in [5], and more recently in [6]; however, spherical calibration approaches are typically not robust w.r.t. noisy image features. The desktop calibration approach proposed in [7] uses a planar (2D) checkerboard to achieve a good trade-off between accuracy and flexibility. The same author proposed in [8] an approach based on linear (1D) artifacts that can be used for simultaneous 
calibration of multiple cameras with a partially overlapping field of view. Another desktop approach appears in [9]: by exploiting the image of two arbitrary coplanar circles, the focal length of the camera and its extrinsic parameters are obtained.

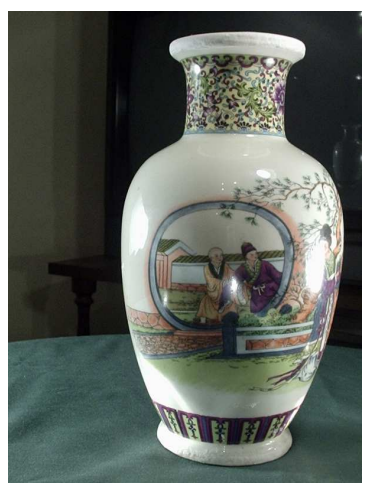

(a)

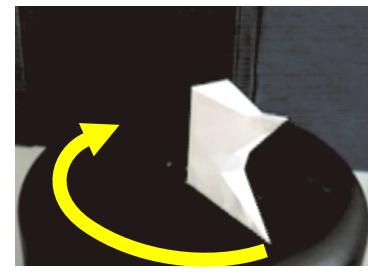

(c)

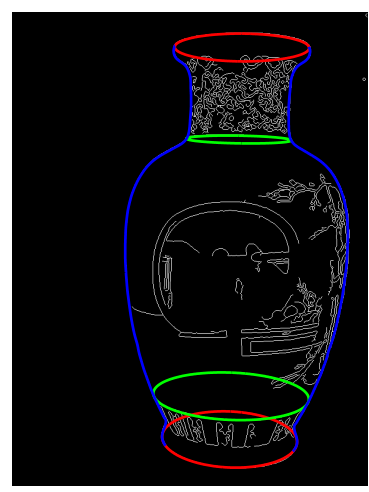

(b)

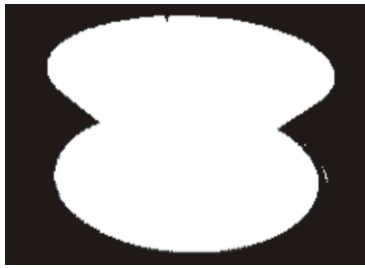

(d)

Fig. 1. (a): A real SOR object. (b): Characteristic curves (apparent contour, imaged cross sections) extracted from (a). (c): An object undergoing Single Axis Motion on a turntable. (d): The virtual SOR induced by the rotating object in (c).

Being quite common in man-made environments, surfaces of revolution (SORs) were also proposed for desktop internal calibration purposes [10] and single view metric reconstruction [11], [12]. Thanks to their symmetry properties, SORs can be conveniently used as multiple camera calibration artifacts. The SOR features usable for calibration are the elliptical imaged cross-sections and the apparent contour. In [10], the apparent contour alone is used to calibrate the camera; this method requires that that two SORs are present in the same image, or that two or more images of SORs taken from the same camera are available. In [12] it is shown that the visible portions of two manually segmented imaged cross-sections are enough for calibrating from one view the focal length and the principal point provided that the camera has square pixels (a constraint always met by the modern devices), even when a single SOR object is present in the image. 
In this paper, we present a desktop calibration approach based on the presence in the image of at least two coaxial circles. Such a geometric configuration often arises in practical applications, either in static scenes of a rotationallysymmetric object or in dynamic scenes of a generic object rotating on a turntable (Single Axis Motion, SAM). A unified framework is provided for both cases, by extracting a SOR object from image data, and using it as calibration artifact. However, while in the former case the SOR is a real object (Fig. 1(a, b)), in the latter case it is actually a "virtual surface," whose image is obtained by superposition of the difference between the current and the first frame of the sequence (Fig. 1(c, d)). As in [12], calibration of a square pixel camera is achieved from a single SOR view. However, that calibration approach is extended here to both internal and external parameters, and is completely automatic, thanks to a homology-based curve segmentation strategy. In addition, our approach combines both the calibration primitives exploited in [12] (imaged cross-sections) and those used in [10] (apparent contour) so as to add robustness and accuracy to the calibration task. Besides, the approach offers a new solution to the problem of camera calibration from turntable sequences, differing from previous solutions (see e.g. [13]) in that it doesn't require point tracking and can also deal with textureless objects. Experimental results provide a quantitative evaluation of calibration performance and demonstrate the use of the approach for the purpose of metric $3 \mathrm{D}$ reconstruction and texture acquisition in practical applications.

\section{Automatic SOR Segmentation}

A SOR can be parameterized as

$$
\mathcal{P}(\vartheta, t)=(\rho(t) \cos (\vartheta), \rho(t) \sin (\vartheta), t),
$$

where $\vartheta \in[0,2 \pi]$ and $t \in[0,1]$. The scaling function $\rho(z)$ controls the 3D shape of the SOR. The perspective projection of a SOR like the vase of Fig. 1(a) gives rise to two different kinds of image curves, namely the apparent contour and the imaged cross sections of Fig. 1(b). The former is the image of the points at which the surface is smooth and the projection rays are tangent to the surface. The shape of this curve is view dependent. On the other hand, imaged cross sections are view independent elliptical curves, which correspond to parallel coaxial circles in 3D and arise from surface normal discontinuities or surface texture content. Both the apparent contour and the imaged cross sections of a SOR are transformed onto themselves by a 4-dof harmonic homology

$$
\mathrm{H}=\mathrm{I}-2 \frac{\mathbf{v}_{\infty} \mathbf{l}_{s}^{\mathrm{T}}}{\mathbf{v}_{\infty}^{\mathrm{T}} \mathbf{l}_{s}},
$$

where $\mathbf{l}_{s}$ and $\mathbf{v}_{\infty}$ are respectively the imaged axis of revolution and the vanishing point of the normal direction of the plane through $\mathbf{l}_{s}$ and the camera center [14].

The SOR segmentation problem concerns with automatically estimating from a SOR image the harmonic homology of Eq. 2 together with the imaged SOR 


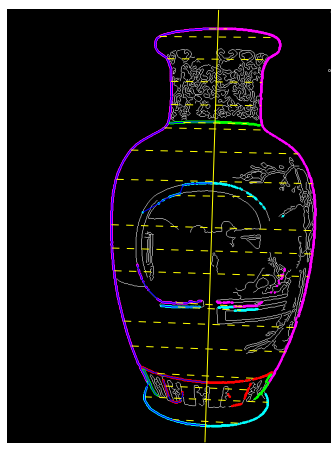

(a)

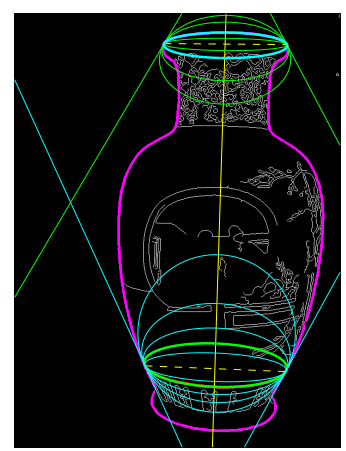

(b)

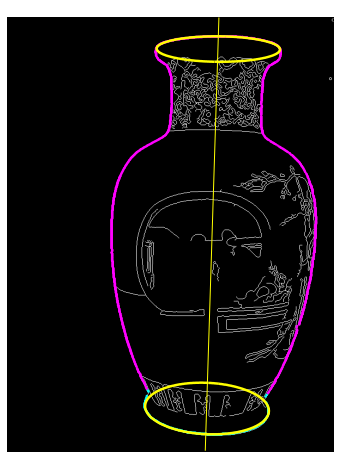

(c)

Fig. 2. Automatic SOR segmentation. (a): Homology estimation and curve segmentation. (b): Conic pencil-based curve classification. (c): The final result.

curves (apparent contour, visible imaged cross sections) consistent with it. All of this geometric information will be exploited later to calibrate the camera. The segmentation strategy follows closely the two-phase approach proposed in [15]. The first phase is devoted to estimating the harmonic homology and all the image curves (possibly including clutter) consistent with it. This is achieved by solving an optimization problem involving edge points extracted from the image according to a multiresolution scheme, where the RANSAC algorithm is used at the lowest resolution level to provide a first guess of the homology parameters. In Fig. 2(a) the final output of the first phase is shown.

The second phase is devoted to classifying the image curves obtained before respectively into (a) apparent contour, (b) imaged cross sections and (c) clutter. To this aim, the tangency condition between each imaged cross-section and the silhouette is exploited, allowing us to construct a conic pencil for each silhouette point pair (Fig. 2(b)), and to look, among all possible conic pencils, for the two ellipses receiving the largest consensus in a Hough-like voting procedure (Fig. 2(c)). Besides being of key importance for the purpose of SOR segmentation, the use of the apparent contour significantly improves the quality of the homology estimate, and hence of the calibration parameters estimated from it.

Automatic segmentation of the imaged virtual SOR arising from SAM sequences follows the same lines as above, but is significantly easier thanks to the fact that clutter is almost absent, and binary images (with the virtual SOR as the foreground) are used, instead of color images.

\section{Camera Calibration}

\subsection{Internal Parameters}

The imaged SOR fixed entities are strictly related to the calibration matrix K, which embeds information about the internal camera parameters. In particular 
it holds $\mathbf{l}_{s}=\omega \mathbf{v}_{\infty}$, where $\omega=\mathrm{K}^{-\mathrm{T}} \mathrm{K}^{-1}$ is referred to as the image of the absolute conic (IAC) [16]. Moreover, since cross sections are parallel circles in 3D, they intersect at the circular points of the families of planes orthogonal to the SOR symmetry axis. Their projection in the image, $\mathbf{i}$ and $\mathbf{j}$, are also related to the image of the absolute conic as $\mathbf{i}^{\mathrm{T}} \omega \mathbf{i}=0$ and $\mathbf{j}^{\mathrm{T}} \omega \mathbf{j}=0$. The resulting system

$$
\left\{\begin{array}{l}
\mathbf{i}^{\mathrm{T}} \omega \mathbf{i}=0 \\
\mathbf{j}^{\mathrm{T}} \omega \mathbf{j}=0 \\
\mathbf{l}_{s}=\omega \mathbf{v}_{\infty}
\end{array}\right.
$$

provides four linear constraints on $\omega$, whose coefficients can be computed from (the visible portions of) two imaged ellipses as shown in [12]. In that paper, it is demonstrated that only three out of the four constraints above are actually independent. Therefore, the system of Eq. 3 can be used to calibrate a square pixel camera (zero skew and unit aspect ratio: 3 dofs) from a single image.

\subsection{External Parameters}

In [17], external orientation is obtained from the imaged cross sections of a right straight homogeneous generalized cylinder (RSHGC) under orthographic viewing conditions. In the following we address the problem of external calibration under full perspective viewing conditions from the image of two cross sections of a SOR - this being a specialization of a RSHGC. Similarly to [18] and [9], our solution is based on the image of two circles, but with the important difference that in our case the circles are coaxial, and not coplanar. Our approach exploits the knowledge of (1) the imaged SOR symmetry axis $\mathbf{l}_{s}$; (2) the vanishing line $\mathbf{l}_{\infty}=\mathbf{i} \times \mathbf{j}$ common to all the planes orthogonal to the SOR symmetry axis, and (3) one or more imaged cross sections. We recall that the matrix K represents only the internal camera parameters; the complete projection matrix is

$$
\mathrm{P}=\mathrm{KR}\left[\mathrm{I}_{3 \times 3} \mid-\mathrm{C}\right],
$$

where the 3 -vector $\mathbf{C}$ is the camera center in (inhomogeneous) world coordinates, and $\mathrm{R}$ is the rotation between the world frame and the camera frame. Without loss of generality, we can take as world frame origin the center of the bottom cross section of the SOR, and as Z axis the SOR symmetry axis; furthermore, we can impose that the camera center must lie on the half plane $\mathrm{X}>0, \mathrm{Y}=0$.

Rotation Matrix. The first step is the computation of the rotation matrix

$$
\mathrm{R}=\left[\begin{array}{lll}
\mathbf{n}_{\mathrm{X}} & \mathbf{n}_{\mathrm{Y}} & \mathbf{n}_{\mathrm{Z}}
\end{array}\right],
$$

where $\mathbf{n}_{\mathrm{x}}, \mathbf{n}_{\mathrm{Y}}, \mathbf{n}_{\mathrm{Z}}$ are unit vectors. It is well known that, given a point image $\mathbf{p}$ in homogeneous coordinates, the inhomogeneous 3 -vector $\mathrm{K}^{-1} \mathbf{p}$ represents the direction (with respect to the camera frame) of the ray passing through the camera center and $\mathbf{p}[16]$. Therefore, if we choose any two points on the line $\mathbf{l}_{s}$, 
we can determine two vectors lying on the plane $\mathrm{Y}=0$, whose normalized cross product provides us with the unit vector $\mathbf{n}_{\mathrm{Y}}$. (The sign of the cross product must be consistent with the definition of the world frame orientation given above - see also the example below.) The same procedure can be applied to compute the unit vector $\mathbf{n}_{\mathrm{Z}}$ from two points properly chosen on the vanishing line $\mathbf{l}_{\infty}$. Finally, the unit vector $\mathbf{n}_{\mathrm{X}}$ is computed as the cross product of $\mathbf{n}_{\mathrm{Y}}$ and $\mathbf{n}_{\mathrm{Z}}$. Fig. 3 shows three points which can be conveniently chosen for obtaining the rotation matrix. These are:

- the homology vertex $\mathbf{v}_{\infty} \in \mathbf{l}_{\infty}$, computed as shown in Section 2;

- the imaged center of the bottom cross section $\mathbf{x}_{c}$. This is the projection of the world origin in the image, and can be obtained from the pole-polar relationship between the imaged bottom cross-section (represented by the $3 \times 3$ symmetric matrix $\mathrm{C}_{b}$ ) and the vanishing line $\mathbf{l}_{\infty}$ as $\mathbf{x}_{c}=\mathrm{C}_{b}^{-1} \mathbf{l}_{\infty}$;

- the intersection $\mathbf{x}_{i}=\mathbf{l}_{s} \times \mathbf{l}_{\infty}$ between $\mathbf{l}_{s}$ and $\mathbf{l}_{\infty}$.

In Fig. 3, the imaged $\mathrm{Z}$ axis $\left(\mathbf{l}_{s}\right)$ is oriented from $\mathbf{x}_{c}$ to $\mathbf{x}_{i}$. Since the $\mathrm{X}$ coordinate of the camera center is positive, the vector

$$
\mathbf{m}_{\mathrm{Y}}=\left(\mathrm{K}^{-1} \mathbf{x}_{c}\right) \times\left(\mathrm{K}^{-1} \mathbf{x}_{i}\right)=\mathrm{K}^{\top}\left(\mathbf{x}_{c} \times \mathbf{x}_{i}\right)
$$

must have the same direction as the $\mathrm{Y}$ axis, in order to obtain a right-hand world frame. The vector $\mathbf{m}_{\mathrm{Z}}$ orthogonal to the plane $\mathrm{Z}=0$ and directed as the $\mathrm{Z}$ axis must then be obtained as

$$
\mathbf{m}_{\mathrm{z}}=\left(\mathrm{K}^{-1} \mathbf{v}_{\infty}\right) \times\left(\mathrm{K}^{-1} \mathbf{x}_{i}\right)=\mathrm{K}^{\top}\left(\mathbf{v}_{\infty} \times \mathbf{x}_{i}\right) .
$$

The unit vectors $\mathbf{n}_{\mathrm{Y}}$ and $\mathbf{n}_{\mathrm{Z}}$ are finally obtained by normalization of $\mathbf{m}_{\mathrm{Y}}$ and $\mathbf{m}_{\mathrm{Z}}$, respectively.

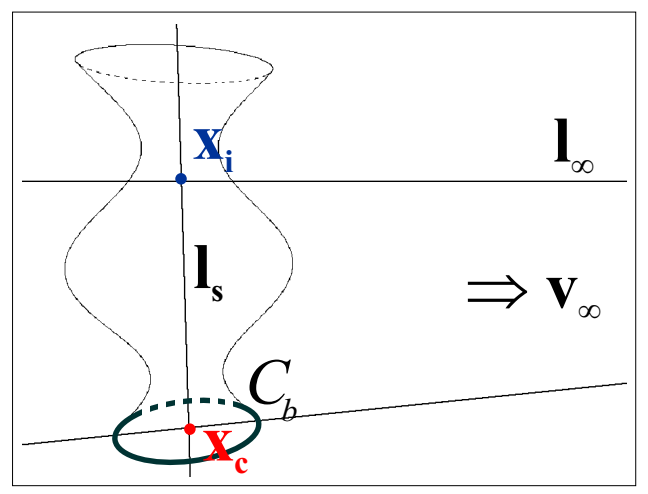

Fig. 3. Lines and points needed for rotation matrix computation.

As the matrix $\mathrm{R}$ thus computed is seldom a rotation matrix, a final refinement step based on the SVD decomposition is carried out to obtain the best orthogonal approximation to $\mathrm{R}[7]$. 
Camera Center. The last step is that of the computation of camera center. Although any visible cross section of known height $\mathrm{z}$ could be exploited, for the sake of simplicity, in what follows we will use the bottom cross section (at $\mathrm{Z}=0$ ), the extension of the equations to the general case being straightforward. Let $\rho$ be the radius of the bottom cross-section, and consider again the projection matrix $\mathrm{P}$. Any point on the plane $\mathrm{Z}=0$ is mapped onto the image by the homography $\mathrm{H}_{0}$ given by:

$$
\begin{aligned}
& \mathbf{x}=\mathrm{P}(\mathrm{X}, \mathrm{Y}, 0,1)^{\top}= \\
& =\left[\begin{array}{lll}
\mathbf{p}_{1} & \mathbf{p}_{2} & \mathbf{p}_{4}
\end{array}\right](\mathrm{X}, \mathrm{Y}, 1)^{\top}= \\
& =\mathrm{H}_{0}(\mathrm{X}, \mathrm{Y}, 1)^{\top} \text {, }
\end{aligned}
$$

where $\mathbf{p}_{i}$ is the i-th column of P. In particular, the center of the bottom cross section is projected onto the inhomogeneous point with pixel coordinates $\left(x_{c}, y_{c}\right)$, whose corresponding homogeneous vector is

$$
\sigma \mathbf{x}_{c}=\sigma\left(\begin{array}{c}
x_{c} \\
y_{c} \\
1
\end{array}\right)=\mathrm{H}_{0}\left(\begin{array}{l}
0 \\
0 \\
1
\end{array}\right)=\mathbf{p}_{4}
$$

More generally, the homography $\mathrm{H}_{0}$ transforms any point of the bottom cross section into the homogeneous image point

$$
\mathbf{x}_{\vartheta}=\mathrm{H}_{0}\left(\begin{array}{c}
\rho \cos \vartheta \\
\rho \sin \vartheta \\
1
\end{array}\right)=\rho \cos \vartheta \mathbf{p}_{1}+\rho \sin \vartheta \mathbf{p}_{2}+\sigma \mathbf{x}_{c},
$$

with pixel coordinates $\left(x_{\vartheta}, y_{\vartheta}\right)$ such that

$$
\frac{y_{\vartheta}}{x_{\vartheta}}=\frac{\rho \cos \vartheta p_{21}+\rho \sin \vartheta p_{22}+\sigma y_{c}}{\rho \cos \vartheta p_{11}+\rho \sin \vartheta p_{12}+\sigma x_{c}}
$$

where $p_{i j}$ denotes the $(i, j)$ element of P. Solving Eq. 10 for $\sigma$, we obtain

$$
\sigma=\frac{\left(p_{21} x_{\vartheta}-p_{11} y_{\vartheta}\right) \cos \vartheta+\left(p_{22} x_{\vartheta}-p_{12} y_{\vartheta}\right) \sin \vartheta}{y_{\vartheta} x_{c}-x_{\vartheta} y_{c}} \rho .
$$

Now, since by definition of the matrix $\mathbf{P}$, the camera center $\mathbf{C}$ appears only in the fourth column:

$$
\mathbf{p}_{4}=-\mathrm{KRC},
$$

by replacing Eq. 12 into Eq. 8 we finally obtain

$$
\mathbf{C}=(\mathrm{KR})^{-1}\left(-\sigma \mathbf{x}_{c}\right)=-\sigma \mathrm{R}^{\top} \mathrm{K}^{-1} \mathbf{x}_{c}
$$

Eqs. 11 through 13 show that, if the real size of the SOR is unknown, its distance w.r.t. the camera can be determined up to an arbitrary scale. Therefore, if the real dimensions of the SOR are not available, the radius $\rho$ can arbitrarily be set to 1. The other parameters involved in Eqs. 11 and 13 can all be computed from the image. Specifically, the imaged world center $\mathbf{x}_{c}$ can be obtained as shown in the previous Section and, for any arbitrarily chosen $\vartheta$, the point $\left(x_{\vartheta}, y_{\vartheta}\right)$ on the imaged cross section can be obtained as shown in [12]. 


\section{Experimental results and applications}

In order to assess the performance of the calibration algorithm, both synthetic and real-world tests were carried out. In the synthetic experiments, the reference SOR view of Fig. 3 was generated, corresponding to the following ground truth camera parameters: $f=750$ (focal length), $\left(x_{p}, y_{p}\right)=(400,300)$ (principal point), $\mathbf{C}=(1.6,0.0,0.7)$ (camera center). Ground truth data were corrupted with increasing Gaussian noise values ranging from 0 to 1.6; for each of these values, 1000 Monte Carlo trials were performed.

Table 1. Calibration performance: focal length and principal point (ground truth: 750 , $(400,300))$.

\begin{tabular}{|c|c|c|c|c|c|c|}
\hline$\sigma$ & $\operatorname{avg}(f)$ & $\operatorname{std}(f)$ & $\operatorname{avg}\left(x_{p}\right)$ & $\operatorname{std}\left(x_{p}\right)$ & $\operatorname{avg}\left(y_{p}\right)$ & $\operatorname{std}\left(y_{p}\right)$ \\
\hline 0.1 & 752.99 & 6.650 & 400.83 & 3.920 & 299.11 & 0.681 \\
0.2 & 749.73 & 7.524 & 399.34 & 4.622 & 300.13 & 0.883 \\
0.4 & 748.53 & 8.770 & 398.90 & 5.388 & 299.96 & 1.138 \\
0.8 & 751.51 & 11.572 & 399.07 & 7.242 & 299.86 & 1.809 \\
1.6 & 744.05 & 15.543 & 394.47 & 9.374 & 301.16 & 3.156 \\
\hline
\end{tabular}

Tab. 1 gives the internal calibration performance (average and standard deviation) for the focal length and principal point. The results show that performance undergoes a graceful degradation as the noise increases. Specifically, the average remains almost constant for all noise values considered, while the standard deviation proportionally increases with noise.

Tab. 2 provides calibration performance for external parameters. Results show that the rotation matrix is more sensitive than the camera center to image noise. Specifically, both the average and standard deviation values of the angle between homologous unit vectors increase with noise. Performance in terms of camera center follows instead the same pattern as with internal parameters, with almost constant average error values, and linearly increasing standard deviation values.

Table 2. External calibration estimates for increasing noise values. Left: Average value and standard deviation of the angle, in degrees, between each column of $\mathrm{R}$ and its estimate. Right: Camera center (ground truth: $\mathrm{x}=1.6, \mathrm{z}=0.7$ ), with $\vartheta=0$.

\begin{tabular}{|c|c|c|c|c|c|c||c|c|c|c|}
\hline & \multicolumn{5}{|c||}{ ROTATION } & \multicolumn{3}{c|}{ CAMERA CENTER } \\
\cline { 2 - 11 }$\sigma$ & $\operatorname{avg}\left(\angle_{\mathrm{X}}\right)$ & $\operatorname{std}\left(\angle_{\mathrm{X}}\right)$ & $\operatorname{avg}\left(\angle_{\mathrm{Y}}\right)$ & $\operatorname{std}\left(\angle_{\mathrm{Y}}\right)$ & $\operatorname{avg}\left(\angle_{\mathrm{Z}}\right)$ & $\operatorname{std}\left(\angle_{\mathrm{Z}}\right)$ & $\operatorname{avg}(\mathrm{X})$ & $\operatorname{std}(\mathrm{X})$ & $\operatorname{avg}(\mathrm{Z})$ & $\operatorname{std}(\mathrm{Z})$ \\
\hline 0.1 & 0.210 & 0.121 & 0.127 & 0.102 & 0.146 & 0.111 & 1.605 & 0.014 & 0.704 & 0.0042 \\
0.2 & 0.250 & 0.173 & 0.144 & 0.134 & 0.185 & 0.148 & 1.598 & 0.015 & 0.700 & 0.0044 \\
0.4 & 0.300 & 0.227 & 0.186 & 0.140 & 0.213 & 0.212 & 1.597 & 0.018 & 0.696 & 0.0054 \\
0.8 & 0.479 & 0.261 & 0.284 & 0.166 & 0.347 & 0.273 & 1.600 & 0.023 & 0.698 & 0.0088 \\
1.6 & 0.675 & 0.346 & 0.419 & 0.295 & 0.455 & 0.349 & 1.583 & 0.033 & 0.696 & 0.0105 \\
\hline
\end{tabular}




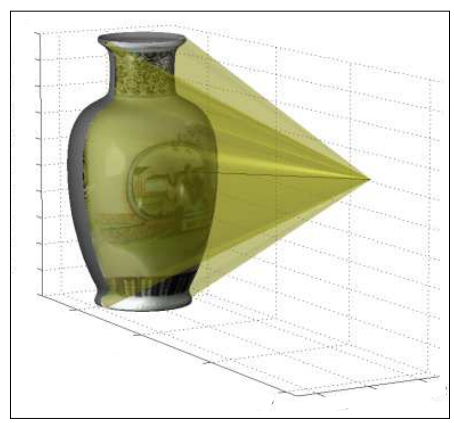

(a)

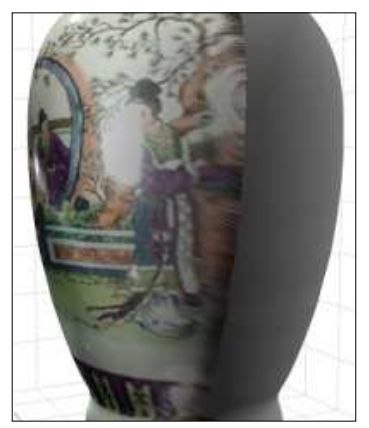

(b)

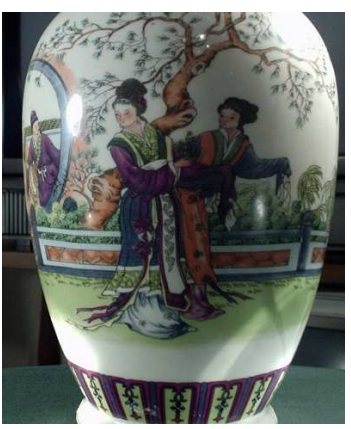

(c)

Fig. 4. (a): The reconstructed camera pose for the vase of Fig. 1(a). (b): A synthetic view of the reconstructed vase. (c): A real photo of the vase from the same viewpoint as in (b).

Real-world tests have concerned texture acquisition of a SOR object, and camera calibration for the SOR and SAM cases. As shown in [12], internal camera calibration permits both the $3 \mathrm{D}$ reconstruction and the texture acquisition of the imaged SOR. However, having computed also the external camera parameters, a much simpler method than the one proposed in that paper can be used to acquire the texture on the SOR. Indeed, for each visible pair $(\vartheta, t)$ in Eq. 1, the corresponding imaged point can be obtained directly via the projection matrix P. In Figs. 4(a,b), the reconstructed camera pose and a synthetic view of the textured model extracted from Fig. 1(a) are shown. The real photo in Fig. 4(c), obtained from the same viewpoint as in (b), confirms the good result obtained, despite the fact that the tree in the original image was highly foreshortened.

Tab. 3(left) reports the ground truth vs estimated values and the error percentage for each of the internal calibration parameters (in pixels) and one external parameter (the third component of the camera center, in $\mathrm{mm}$ ). The ground truth was computed with a $3 \mathrm{D}$ calibration grid and the standard Tsai algorithm [1] — the camera had a negligible radial distortion and square pixels. A similar test

Table 3. Calibration with a real SOR object (left) and with a turntable (SAM) sequence (right). Two different cameras were used. Ground truth and estimated values are denoted respectively as $v$ and $\hat{v}$. The percentage error $\varepsilon_{\%}$ is evaluated as $100 \cdot|v-\hat{v}| / v$.

\begin{tabular}{|c|c|c|c||c|c|c|}
\hline \multirow{2}{*}{ parameter } & \multicolumn{3}{|c||}{ SOR } & \multicolumn{3}{c|}{ SAM } \\
\cline { 2 - 7 } & $v$ & $\hat{v}$ & $\varepsilon \%$ & $v$ & $\hat{v}$ & $\varepsilon \%$ \\
\hline$f$ (focal length) & 718.52 & 728.67 & 1.41 & 398.46 & 390.17 & 2.08 \\
$x_{p}$ (principal point) & 320.01 & 343.27 & 7.27 & 167.22 & 186.62 & 11.60 \\
$y_{p}$ (principal point) & 239.96 & 240.65 & 0.29 & 121.07 & 98.06 & 19.01 \\
$\mathrm{Z}$ (camera center) & 217.82 & 198.26 & 8.98 & 240.01 & 217.37 & 9.43 \\
\hline
\end{tabular}


was conducted for the case of a turntable sequence. Tab. 3(right) shows the comparison between the calibration results obtained by using, as calibration artifact, the virtual SOR segmented as in Fig. 5(a), with those obtained with the Tsai algorithm. For both the real cases addressed, results show a similar performance as for the noise sensitivity of the internal calibration parameters. Specifically, the principal point is more sensitive w.r.t. noise than the focal length. This may be explained by the fact, reported in the literature on SOR-based calibration (see e.g. [12]), that the accuracy of the principal point (but not that of the focal length) depends not only on image noise, but also on the relative position of the imaged SOR axis w.r.t. the principal point itself. In particular, the estimation uncertainty is bigger and bigger as imaged axis of symmetry get closer to the principal point.

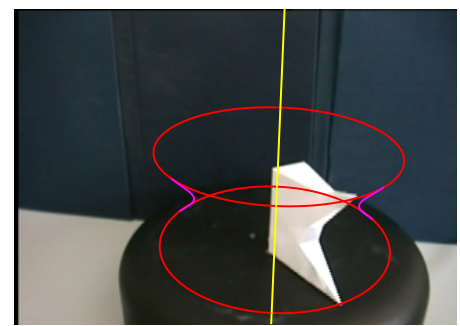

(a)

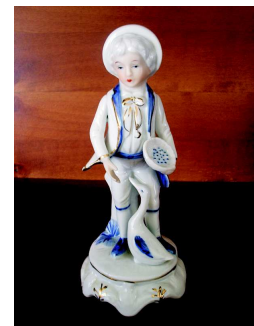

(b)

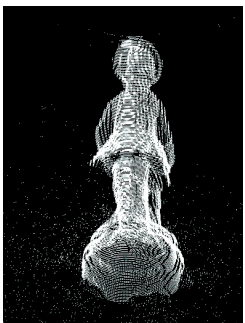

(c)

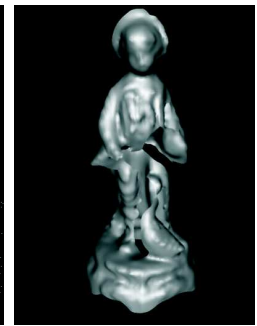

(d)

Fig. 5. (a): The segmented virtual SOR for the object rotating on a turntable. A medium-profile analog camera was used. (b): A complex object. (c,d): The 3D model (point cloud, solid) extracted from a turntable sequence of the object in (b).

Figs. 5(b,c,d) show the reconstruction results for a complex object obtained with a desktop $3 \mathrm{D}$ scanning system based on the calibration procedure described in this paper. The system is composed by a turntable, an square pixel camera and a laser stripe illuminator, which makes visible a vertical slice of the rotating object being acquired. After virtual SOR image extraction and camera calibration performed by exploiting the same object being scanned, shape acquisition is finally obtained by laser profile rectification and collation, as shown in [19]. The $3 \mathrm{D}$ model accurately reproduces the shape of the original object.

\section{Conclusions and future work}

A novel approach was proposed to automatically extract SOR-related image primitives and calibrate both internal and external camera parameters from coaxial circles, arising either from a single image of a SOR or from a turntable sequence featuring arbitrarily-shaped objects. The method has been employed 
successfully in a desktop 3D laser scanner based on SAM and laser profile rectification, obtaining good results.

Although the external calibration approach proposed is view-dependent (the $\mathrm{X}$-axis of the world frame being required to lie on the plane through the SOR axis and the camera center), such 1-parameter dependence can be easily removed given an identifiable reference point on either coaxial circle. Absolute external calibration can be useful for the relative positioning of any pair of cameras having the SOR and the reference point in their fields of view (see Fig. 6).

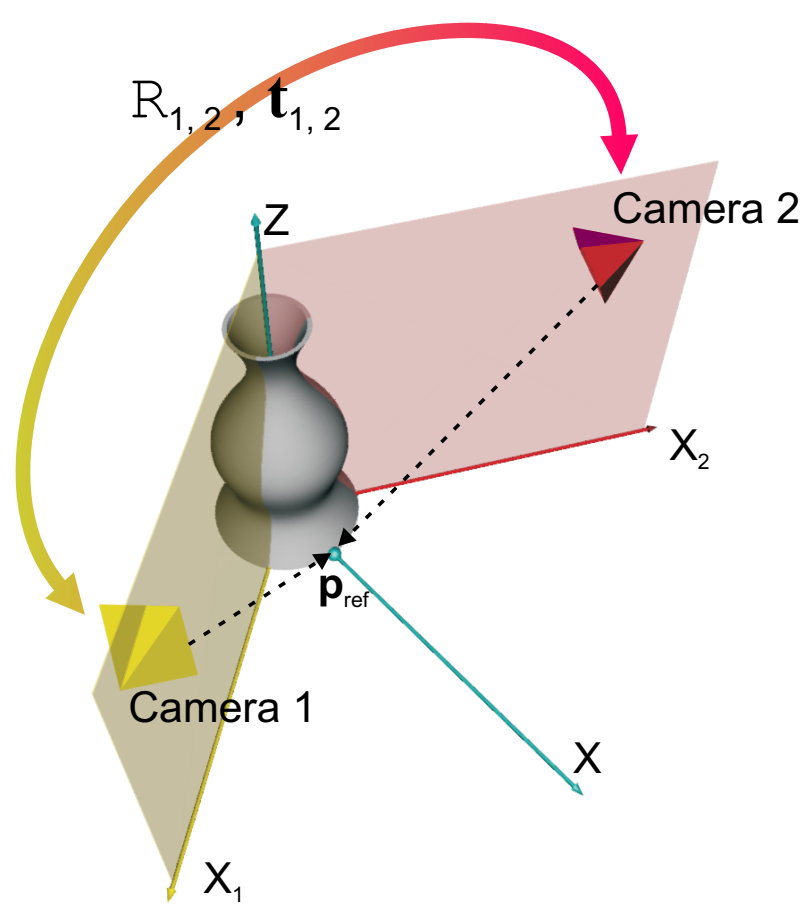

Fig. 6. View-dependent external calibration can be made view-independent using a common reference point $\mathbf{p}_{\text {ref }}$, thus making it possible to compute the relative positioning transformation $\mathrm{R}_{12}, \mathbf{t}_{12}$. The $\mathrm{x}_{i}$ 's are the view-dependent world axes, while $\mathrm{X}$ through $\mathbf{p}_{\text {ref }}$ is the absolute one.

As future work, we are trying to obtain 3D textured models of generic objects, extending the projection method developed for the SOR case. The idea is to project the model point cloud (obtained with the laser scanner) onto each frame of a video sequence of the real object undergoing SAM, after having registered frame by frame the projected point cloud with the blob (obtained by background subtraction) of the rotating object. 


\section{References}

1. Tsai, R.Y.: A versatile camera calibration technique for high-accuracy 3D machine vision metrology using off-the-shelf TV cameras and lenses. IEEE Journal of Robotics and Automation 3 (1987) 323-344

2. Caprile, B., Torre, V.: Using vanishing points for camera calibration. The International Journal of Computer Vision 4 (1990) 127-140

3. Sturm, P., Maybank, S.: A method for interactive 3D reconstruction of piecewise planar objects from single images. In: British Machine Vision Conference. (1999) $265-274$

4. Liebowitz, D., Criminisi, A., Zisserman, A.: Creating architectural models from images. In: EuroGraphics. Volume 18. (1999) 39-50

5. Daucher, N., Dhome, M., Lapreste, J.: Camera calibration from spheres images. In: 3rd European Conference on Computer Vision. (1994) 449-454

6. Agrawal, M., Davis, L.S.: Camera calibration using spheres: A semi-definite programming approach. In: 9th IEEE International Conference on Computer Vision. Volume 2. (2003) 782-789

7. Zhang, Z.: A flexible new technique for camera calibration. IEEE Transactions on PAMI 22 (2000) 1330-1334

8. Zhang, Z.: Camera calibration with one dimensional objects. In: 7th European Conference on Computer Vision. Volume IV. (2002) 161-174

9. Chen, Q., Wu, H., Wada, T.: Camera calibration with two arbitrary coplanar circles. In: European Conference on Computer Vision. (2004) 521-532

10. Wong, K.Y.K., Mendonça, P., Cipolla, R.: Camera calibration from surfaces of revolution. IEEE Transactions on PAMI 25 (2003) 147-161

11. Wong, K.Y.K., Mendonça, P.R.S., Cipolla, R.: Reconstruction of surfaces of revolution from single uncalibrated views. In: British Machine Vision Conference. Volume 1. (2002) 93-102

12. Colombo, C., Del Bimbo, A., Pernici, F.: Metric 3D reconstruction and texture acquisition of surfaces of revolution from a single uncalibrated view. IEEE Transactions on PAMI 27 (2005) 99-114

13. Jiang, G., Quan, L., Tsui, H.T.: Circular motion geometry by minimal 2 points in 4 images. In: IEEE International Conference on Computer Vision. (2003) 221-227

14. Abdallah, S.M.: Object Recognition via Invariance. PhD thesis, The University of Sydney, Australia (2000)

15. Colombo, C., Comanducci, D., Del Bimbo, A., Pernici, F.: Accurate automatic localization of surfaces of revolution for self-calibration and metric reconstruction. In: IEEE CVPR Workshop on Perceptual Organization in Computer Vision. (2004) (On CD-ROM.)

16. Hartley, R.I., Zisserman, A.: Multiple View Geometry in Computer Vision. Cambridge University Press (2000)

17. Xu, G., Tanaka, H.T., Tsuji, S.: Right straight homogeneous generalized cylinders with symmetric cross-sections: Recovery of pose and shape from image contours. In: IEEE International Conference on Computer Vision and Pattern Recognition. (1992) 692-694

18. Rothwell, C.A., Zisserman, A., Marinos, C.I., Forsyth, D., Mundy, J.L.: Relative motion and pose from arbitrary planar curves. Image and Vision Computing 10 (1992) 251-262

19. Colombo, C., Comanducci, D., Del Bimbo, A.: A desktop 3D scanner exploiting rotation and visual rectification of laser profiles. In: IEEE International Conference on Vision Systems. (2006) (On CD-ROM.) 